\title{
TG04
}

\section{Feasibility of Underbalance Drilling in Tight Gas Reservoirs - A New Approach}

\author{
T. Finkbeiner* (GeoMechanics Int.), S. Perumalla (GeoMechanics Int.), D. \\ Moos (GeoMechanics Int.), M. Brudy (GeoMechanics Int.) \& P. Peska \\ (GeoMechanics Int.)
}

\section{SUMMARY}

Deep, tight reservoirs face significant appraisal and development challenges. In particular, it can be difficult proving the presence and mobility of sufficient quantities of gas to make the reservoir economically viable. At the same time, drilling costs are extremely high. In this context, underbalanced drilling (UBD) provides a number of benefits: first, it enables the operator to proof (i.e., provide physical evidence for) the presence of producible quantities of gas (so-called testing while drilling ) while the well is being drilled. Underbalanced drilling also can minimize formation damage and maximize the rate of penetration. This, combined with reduced use of expensive mud formulations, can result in significant savings of drilling and completion costs relative to conventional drilling. However, not all reservoirs are suitable for UBD as there is much greater risk of mechanical wellbore instabilities relative to wells drilled overbalanced. We present a new, realistic approach that enables to increase the accuracy of predictions and at the same time takes scale as well as time depenent effects into consideration. The results of this type of analysis can provide an invaluable help for tight gas reservoir exploration and production. 
Deep, tight reservoirs face significant appraisal and development challenges. In particular, it can be difficult proving the presence and mobility of sufficient quantities of gas to make the reservoir economically viable. At the same time, drilling costs are extremely high. In this context, underbalanced drilling (UBD) provides a number of benefits: first, it enables the operator to proof (i.e., provide physical evidence for) the presence of producible quantities of gas (so-called "testing while drilling") while the well is being drilled. Underbalanced drilling also can minimize formation damage and maximize the rate of penetration. This, combined with reduced use of expensive mud formulations, can result in significant savings of drilling and completion costs relative to conventional drilling. However, not all reservoirs are suitable for UBD as there is much greater risk of mechanical wellbore instabilities relative to wells drilled overbalanced. This is because in overbalanced wells in which the mud pressure on the wellbore wall is greater than the pore pressure, the extra support which is missing when a well is drilled underbalanced) keeps the amount of wellbore failure due to the wellbore stress concentration to manageable levels. Hence, geomechanical analyses prior to drilling are of particular importance in order to evaluate the feasibility of UBD operations.

In the past, the stability of UBD wells has been analyzed using conventional approaches, simply by extending these to stress states in which immediately after the well is drilled one effective principal stress (the radial stress) is tensile; undrained conditions are assumed to develop instantaneously at the wellbore. These models implicitly assume that the well is formed instantaneously in rock with undisturbed pore pressure. The subsequent time-dependent response of the well is then computed using poroelastic models. This approach leads to very conservative predictions of the feasibility of underbalanced drilling, with the result that many wells that would be candidates for UBD are drilled overbalanced.

To apply a less conservative approach, a new analytical model to predict the stability of underbalanced wells has been developed. Based on the recognition that rocks have scale-dependent strengths, the full stress concentration is not developed until some time after the bottom of the well is some distance below the point of interest, and that fluid flow into the advancing wellbore leads to a zone of locally lower pore pressure that extends beneath the drill-bit, it provides rapid assessments of the limit of safe underbalance as a function of drilling rate. The model predicts the regions within which spalling (tensile failure) and breakout (compressive failure) will occur. One consequence is that higher permeability leads to the ability to drill both faster and with a larger underbalance. A second is that smaller hole sizes are predicted to be easier to drill underbalanced; in cases where there is a high risk of wellbore collapse of the full-sized well this suggests that drilling an initial pilot well followed by enlargement to full size may mitigate the risk of collapse. Figure 1 shows an output example indiacting the amount of underbalance a deep, tight gas reservoir is feasible to be drilled for a given range of compressive rock strength; it also shows the amount of failure expected (i.e., breakout width) and where in the well the failure is expected (i.e., on the sides in this case). The results of this example indicate that UBD is feasible with an appreciable amount of underbalance, but some finite failure is expected, which needs to be considered for hole cleaning and hydraulics calculations. 


\section{EAGE}
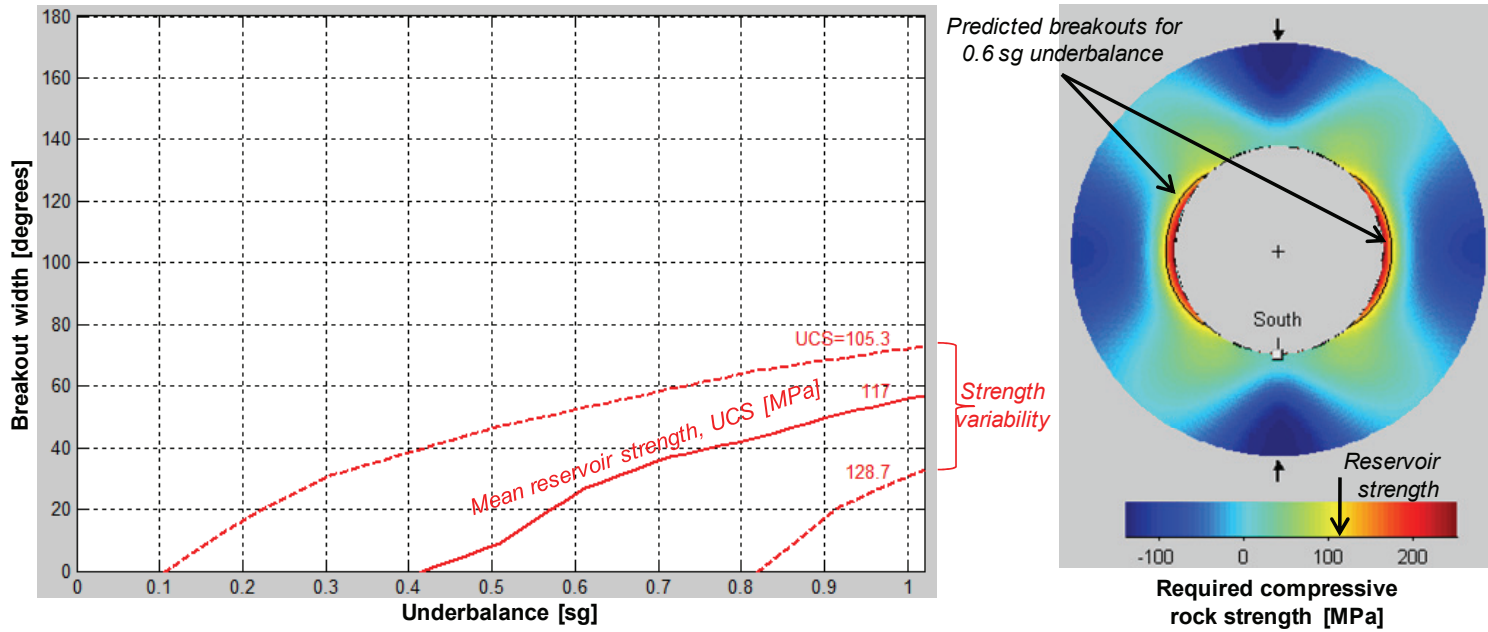

Figure 1 Results from a case study illustrating that UBD is feasible. The plots illustrate predicted breakout width as a function of rock strength and underbalance (left) as well as predicted breakouts and their location around the well for a given rock strength value (right). 